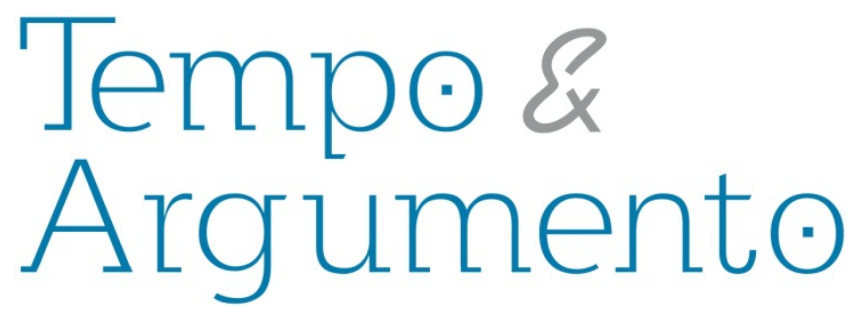

\title{
Calidad educativa y didáctica de la historia en los gobiernos neoconservadores en México 2000-2012
}

\begin{abstract}
Resumen
El presente artículo trabaja sobre el impacto de la calidad educativa en la enseñanza de la historia en México durante los gobiernos del conservador Partido Acción Nacional. En él se describe cómo los criterios de eficacia y relevancia son determinantes para ir transformando la epistemología del conocimiento histórico escolar. El primero lo hace a partir de evaluaciones nacionales e internacionales que obligan a diseñar contenidos históricos en forma de estándares claramente mensurables, secuenciales y que permitan la comparación internacional. El segundo lo hace a través del concepto de competencia y de las habilidades en que se tiene que formar un sujeto cognoscente universal. Asimismo, se explica el papel que jugaron diferentes especialistas en didáctica de la historia para producir esta mutación. Se concluye que a pesar de ciertos avances en las propuestas gubernamentales de enseñanza de la historia, la calidad educativa y la idea de sujeto universal son profundamente excluyentes con otras formas de pensar el pasado dentro de la escuela. Se utilizan como fuentes para el análisis los programas educativos nacionales de los dos gobiernos neoconservadores estudiados, diferentes materiales didácticos y los programas de estudio de las reformas a la educación básica de 2006 y 2011.
\end{abstract}

Palabras clave: Calidad educativa; Enseñanza de la historia; Educación histórica; Pensar históricamente; México.

\section{Sebastián Plá}

Doctor en Pedagogía. Profesor de Asignatura "A" Interino. Universidad

Nacional Autónoma de México sebastianpla@gmail.com

\section{Para citar este articulo:}

PLÁ, Sebastián. Calidad educativa y didáctica de la historia en los gobiernos neoconservadores en México 2000-2012. Revista Tempo e Argumento, Florianópolis, v. 6, n. 11, p. 162-192, jan./abr. 2014.

DOI: $10.5965 / 2175180306112014162$

http://dx.doi.org/10.5965/2175180306112014162 


\section{Educational Quality and history teaching in the neoconservative governments in Mexico 2000-2012}

\begin{abstract}
This article analyzes the impact of educational quality in the teaching of history in Mexico during the administrations of the conservative National Action Party. It describes how the criteria of effectiveness and relevance are crucial to transform the epistemology of historical knowledge in school. The first creates national and international assessments requiring designing curricula as clearly measurable, sequential and allowing international comparison standards. The second does so through the concept of competence and skills by which a universal knowing subject has to be formed. Also, the role played by different researchers in history teaching to produce this mutation is studied. I conclude that despite some progress in government proposals for history teaching, the quality of education and the idea of universal subject are deeply exclusive with other ways of thinking about the past inside the school. I use as sources for analyzing national educational programs, different teaching materials and curricula reforms for basic education, in 2006 and 2011.
\end{abstract}

Keywords: Educational Quality; History Teaching; Historical Thinking; Mexico.

\section{Qualidade educativa e ensino da história nos governos neoconservadores no México (2000-2012)}

\begin{abstract}
Resumo
O presente artigo discute o impacto da qualidade educativa no ensino da história no México durante os governos do partido conservador Accion Nacional. Nele se descreve como os critérios de eficiência e relevância são determinantes para transformar a epistemologia do conhecimento histórico escolar. O primeiro o faz a partir de avaliações nacionais e internacionais que obrigam a estabelecer conteúdos históricos na forma de padrões claramente mensuráveis, sequenciais e que permitam a comparação internacional. O segundo o faz através do conceito de competência e das habilidades em que se tem que formar um sujeito cognoscente universal. Contudo, explica-se o papel que desempenharam diferentes especialistas em ensino da história para produzir esta mudança. Conclui-se que, apesar de certos avanços nas propostas governamentais de ensino da história, a qualidade educativa e a ideia de sujeito universal são profundamente excludentes em relação a outras formas de pensar o passado dentro da escola. Utilizam-se como fontes para a análise os programas educativos nacionais dos dois governos neoconservadores estudados, diferentes materiais didáticos e os programas de estudo das reformas na educação básica de 2006 a 2011.
\end{abstract}

Palavras-chave: Qualidade educativa; Ensino de história; Educação histórica; México. 


\section{Introducción}

México inició su historia política en el siglo XXI con un cambio en apariencia trascendental. Tras setenta y un años de gobierno de un solo partido político en el poder ${ }^{1}$ se dio por primera vez la alternancia en la presidencia de la república. El neoconservador ${ }^{2}$ Partido Acción Nacional (PAN) ganó las elecciones del año 2000 y conservó el poder en las elecciones de 2006, hasta que seis años después, tras doce años de decrecimiento económico y del aumento acelerado de la violencia criminal y de Estado, perdió los comicios y facilitó el retorno del Partido Revolucionario Institucional (PRI) al gobierno federal. $^{3}$ La alternancia en el poder trajo consigo vientos esperanzadores para la democracia mexicana, sin embargo, los cambios en el ámbito político no implicaron modificaciones significativas en los principios rectores de la economía y la educación. Se continuó con el desmantelamiento del frágil estado benefactor y corporativo que caracterizó al sistema político mexicano a lo largo de casi todo el siglo XX y se consolidaron los tres pilares de las políticas neoliberales: privatización de las paraestatales, reducción del gasto público y liberalización de los mercados. El cambio de partido político en el poder renovó ciertos mecanismos de control y privilegió otros aspectos de la vida social, política y económica del país, pero no varió de fondo las políticas económicas ni educativas.

\footnotetext{
1 En 1929 se fundó como partido de Estado el Partido Nacional Revolucionario (PNR), en 1938 cambió su nombre a Partido de la Revolución Mexicana (PRM) y en 1946 tomó el nombre que lleva hasta el día de hoy, Partido Revolucionario Institucional (PRI). Al ser un partido de Estado, surgido del consenso y la coerción entre diferentes grupos de la Revolución Mexicana (1910-1920), logró permanecer en el poder de 1929 a 2000. En 1992 el PRI ya había perdido su tradición revolucionaria y estaba dirigido fundamentalmente por un grupo de tecnócratas. Actualmente, tras las elecciones de 2012, el PRI retornó al poder, ahora bajo el liderazgo de Enrique Peña Nieto.
}

${ }^{2}$ Entiendo en el presente trabajo por neoconservador al pensamiento político que en lo social defiende la estructura patriarcal de la sociedad, es católico y es por lo general muy coercitivo con las libertades individuales. Por el contrario, en el ámbito económico, son neoliberales radicales, por lo que proponen reducción del gasto social, el desarrollo del libre mercado y la privatización de las paraestatales. En cierta medida podría ser usado el término de Michel Apple (2002) de neo-neo, sin embargo, creo que la diferencia fundamental entre Estados Unidos de América y México, es que el segundo son el mismo grupo, mientras que en el primero, a pesar de que en ocasiones están estrechamente vinculados, en principio se encuentran separados. Esto no implica que en México no existan neoliberales que estén distanciados de los neoconservadores

${ }^{3}$ Los dos presidentes panistas fueron Vicente Fox Quesada (2000-2006) y Felipe Calderón Hinojosa (20062012) 
En el ámbito educativo, entre 2000 y 2012 se hicieron importantes reformas a la educación básica y a las escuelas normales -instituciones responsables de la formación de profesores de primaria y secundaria- que produjeron renovados discursos educativos. En 2002 se declaró obligatoria la educación preescolar (3 a 6 años de edad), en 2006 se publicaron nuevos programas para la educación secundaria (12 a 15 años de edad) y en 2009 se inició el proceso denominado Reforma Integral a la Educación Básica en México que concluyó en el año 2011 con nuevos enfoques y contenidos para preescolar, primaria y secundaria. ${ }^{4}$ También en ese año se realizó la reforma a la licenciatura en educación primaria. Parte central de las reformas se hicieron bajo los criterios de calidad educativa que -a pesar de encontrar sus raíces en los años ochenta y noventa, como consecuencia del programa de Educación para Todos (EFA por sus siglas en inglés) - $^{5}$ cobró notoria fuerza en el siglo XXI bajo los gobiernos de derecha. Dos ejemplos de lo anterior son la firma de los acuerdos políticos con el Sindicato Nacional de Trabajadores de la Educación (SNTE) denominados Compromiso Social por la Calidad de la Educación de 2002 y la Alianza para la Calidad Educativa de 2008. La calidad educativa vino de la mano con un férreo sistema de evaluación docente para la educación básica, en el que destacan la fundación del Instituto Nacional de Evaluación Educativa (INEE) en 2002, dispositivo central para la gobernación del sistema educativo mexicano y la creación del examen censal denominado Evaluación Nacional del Logro Académico en Centros Escolares (ENLACE) en 2006.

\footnotetext{
${ }^{4}$ El sistema educativo mexicano ha sufrido importantes reestructuraciones de 1992 a la fecha. Actualmente, su columna vertebral está compuesta por el preescolar, la primaria y la secundaria (3 a 15 años) y juntos conforman la educación básica y obligatoria. El bachillerato (15 a 18) años es obligatorio pero no es educación básica. Finalmente se encuentra la educación superior. Dentro de cada uno de los niveles hay subsistemas muy variados, así como instituciones dedicadas a la educación indígena o a sectores marginados de la población. Sin embargo, dada las dimensiones del sistema educativo mexicano, aquí solo trabajo con el núcleo central, la educación básica y obligatoria y la formación de maestros para estos niveles educativos.

${ }^{5}$ Education for All (EFA) es quizá el proyecto educativo más importante de la UNESCO de los últimos veinticinco años. Su objetivo es una educación de calidad con equidad para satisfacer las necesidades básicas de aprendizaje, es decir, "Estas necesidades abarcan tanto las herramientas esenciales para el aprendizaje (como la lectura y la escritura, la expresión oral, el cálculo, la solución de problemas) como los contenidos básicos del aprendizaje (conocimientos teóricos y prácticos, valores y actitudes) necesarios para que los seres humanos puedan sobrevivir, desarrollar plenamente sus capacidades, vivir y trabajar con dignidad, participar plenamente en el desarrollo, mejorar la calidad de su vida, tomar decisiones fundamentadas y continuar aprendiendo" (UNESCO, 1990, p. 3)
} 
Las reformas curriculares de estos dos sexenios y la instalación de las primeras bases de un sistema nacional de evaluación ${ }^{6}$ mudaron los contenidos y las didácticas escolares que, a pesar de tener parte de sus cimientos en la reforma educativa de 1992, encontraron nuevas formas de expresión a partir de por lo menos tres elementos: la formación por competencias, los estándares curriculares y los aprendizajes esperados. La consecución efectiva de estos tres elementos será parte constitutiva de los que se propone como calidad educativa. Por tanto, y con base en lo anterior, me surgen tres preguntas que trataré de responder en este escrito: ¿Cuál es la influencia de los proyectos de calidad educativa y de evaluación internacionales en los programas de historia para la educación básica en México? ¿Cómo se conforma la nueva epistemología del conocimiento histórico escolar? y por último ¿qué papel juega la didáctica de la historia en el proceso de inclusión/exclusión de saberes y sujetos en las reformas educativas neoconservadores en México entre 2000 y 2012? Para dar respuesta a esta pregunta he dividido mi reflexión en cuatro partes. En la primera describo la calidad educativa como idea fuerza de los proyectos educativos conservadores en México. Posteriormente, delineo el lugar que ocupa la asignatura de historia dentro del mapa curricular para la educación básica. En tercer lugar me concentro en la epistemología del conocimiento histórico escolar prescita en los programas, con especial énfasis en el concepto de competencia histórica. Por último analizo el papel de la didáctica de la historia y sus actores en la conformación de un saber histórico basado en la idea de calidad educativa. El objetivo es demostrar cómo la propuesta de calidad educativa y sus manifestaciones en saberes escolares, en este caso la historia, propone la idea de un sujeto cognoscente universal profundamente excluyente de otras formas culturas de construir conocimiento. Esta idea es resultado de gobiernos conservadores en lo social y neoliberales en lo económico.

Vale la pena aclarar que dado que en el presente texto mis fuentes son fundamentalmente documentos publicados por la Secretaría de Educación Pública (SECRETARÍA DE EDUCACIÓN PÚBLICA), mi trabajo se basa en las propuestas oficiales creadas desde el poder, lo que no implica necesariamente que el impacto de las reformas

\footnotetext{
${ }^{6}$ Este sistema quedó finalmente plasmado en la constitución política en 2013.
} 
se esté desarrollando en las aulas tal cual lo planeado y tampoco asevero que no haya existido resistencia alguna a estas transformaciones educativas en general y a la propia enseñanza de la historia. Por tanto, reconozco las limitaciones y alcances de mi propio análisis a la dimensión prescriptiva de la enseñanza de la historia y a mi imposibilidad de poder ver sus múltiples manifestaciones en las aulas.

\section{Calidad educativa}

Calidad educativa es un concepto poderoso. Su carácter difuso y difícil de asir le da la posibilidad de adaptarse a diferentes circunstancias nacionales sin modificar sus principios universalistas. En México, como en otras partes del mundo, desde finales del siglo XX se han imbricados dos propuestas diferentes pero no antagónicas sobre calidad educativa. Estas son la mirada economicista esgrimida principalmente por el Banco Mundial (BM) y la corriente humanística y más próxima al desarrollo humano sostenida por la Organización de las Naciones Unidas para la Educación, la Ciencia y la Cultura (UNESCO). La perspectiva del BM se basa en el estudio de productos cuantitativos que puedan medir la calidad, por ejemplo, las tasas de retorno de la inversión en educación y el rendimiento cognitivo, medido a través de grandes pruebas o exámenes nacionales e internacionales. Por otro lado, la mirada de la UNESCO se centra más en los procesos educativos y sus resultados para el desarrollo sostenible, las políticas de inclusión educativa, la alfabetización y el aprendizaje de habilidades cognitivas básicas, lo que no implica estar en contra de ciertas mediciones generales o globales (BARRET, 2006, pp. 23). A pesar de las divergencias de objetivos, de los diferentes motivos que las impulsan y de sus prioridades educativas, se pueden mencionar cinco principios relativamente consensuados que definen la calidad educativa: eficiencia, eficacia, equidad, pertinencia y relevancia. ${ }^{7}$ Como veremos más adelante, aunque los conceptos de pertinencia y

\footnotetext{
${ }^{7}$ De manera sucinta podemos definir cada componente. Eficiencia: la capacidad para usar el menor número de insumos para alcanzar el objetivo deseado. Eficacia: la capacidad de alcanzar los niveles de logro de los objetivos planteados. Equidad: mayor apoyo a los grupos denominados marginados para igualar las condiciones de la competencia. Relevancia: Desarrollo de aprendizajes significativos para el desenvolvimiento productivo en la sociedad y para el desarrollo personal. Pertinencia: La educación debe responder a las condiciones personales y socioculturales del estudiante. Estos conceptos no son los únicos pero son los más utilizados. Asimismo existen entre ellos muchas tensiones difíciles de
} 
relevancia son en principio los que más afectan a los saberes escolares, la eficacia a través de la evaluación también termina siendo un determinante.

A pesar de que el concepto de calidad educativa y su casi natural adjetivo de equidad se sedimentó en la jerga política pedagógica mexicana en la última década del siglo $\mathrm{XX}$, no es hasta los gobiernos conservadores y empresariales de Vicente Fox Quesada y Felipe Calderón Hinojosa que ocupa el centro desde el cual se establece toda clasificación y jerarquización del discurso educativo. El Plan Sectorial de Educación 20012006 llevaba consigo la reinterpretación mexicana del EFA: Por una educación de buena calidad para todos. Un enfoque educativo para el siglo XXI que marcó las directrices para la creación del Programa de Escuelas de Calidad (PEC), facilitó en 2002 el uso del concepto de calidad educativa en la constitución política mexicana y la firma de Compromiso Social por la Calidad de la Educación, acuerdo político que reunió a las autoridades educativas, al SNTE, a grupos empresariales, religiosos y ultraconservadores de la sociedad. El principal objetivo del Compromiso fue ofrecer a la sociedad mexicana "una educación de buena calidad, que permita a los niños y niñas mexicanos alcanzar los más altos estándares de aprendizaje, sin distinción de género, etnia o condición social” (MÉXICO, 2002). Para velar por la calidad educativa se creó el 8 de agosto de 2002 el Instituto Nacional de Evaluación Educativa (INEE), ya que la calidad de la educación, "requiere de un sistema de evaluación sólido, confiable, oportuno y transparente, cuyos resultados puedan satisfacer la demanda social por conocer los resultados del sistema educativo nacional y fortalecer el proceso de toma de decisiones" (SECRETARÍA DE EDUCACIÓN PÚBLICA, 2002, p. 1). En esta etapa, todavía se puede ver el predominio de la mirada humanista de la UNESCO, aunque no excluye el lente del BM y los estándares de aprendizaje. En la definición de calidad educativa para educación básica del gobierno de la república:

Una educación básica de buena calidad está orientada al desarrollo de las competencias cognoscitivas fundamentales de los alumnos, entre las que destacan las habilidades comunicativas básicas, es decir, la lectura, la escritura, la comunicación verbal y el saber escuchar.

Una educación básica de buena calidad debe formar en los alumnos el interés y la disposición a continuar aprendiendo a lo largo de su vida, de

resolver, por ejemplo eficiencia y equidad son en muchas ocasiones difíciles de compatibilizar, pero este problema desborda el objetivo de mi investigación. 
manera autónoma y autodirigida; a transformar toda experiencia de vida en una ocasión para el aprendizaje.

Una educación básica de buena calidad es aquella que propicia la capacidad de los alumnos de reconocer, plantear y resolver problemas; de predecir y generalizar resultados; de desarrollar el pensamiento crítico, la imaginación espacial y el pensamiento deductivo.

Una educación básica de buena calidad brinda a los alumnos los elementos necesarios para conocer el mundo social y natural en el que viven $\mathrm{y}$ entender éstos como procesos en continuo movimiento $\mathrm{y}$ evolución.

Una educación básica de buena calidad proporciona las bases para la formación de los futuros ciudadanos, para la convivencia y la democracia y la cultura de la legalidad.

En una educación básica de buena calidad el desarrollo de las competencias básicas y el logro de los aprendizajes de los alumnos son los propósitos centrales, son las metas a las cuales los profesores, la escuela y el sistema dirigen sus esfuerzos (MÉXICO, 2001, p. 123).

Los elementos centrales de esta propuesta de calidad provienen claramente del EFA: desarrollo de competencias, centralidad de las habilidades comunicativas, nociones de aprender a lo largo de la vida, resolución de problemas y la centralidad del aprendizaje entre otros. Son los conceptos de pertinencia y relevancia los que parecen tener más fuerza. Es decir, lo que se impone bajo esta noción de calidad es la idea de un sujeto cognoscente y trabajador universal que posee "competencias cognoscitivas fundamentales”. El INEE y el nuevo sistema de evaluación educativa tenían que apegarse a esta noción de de calidad. Asimismo, los contenidos curriculares entre ellos la historia, también se verían obligados a adaptarse para sobrevivir.

Durante el segundo sexenio neoconservador, el concepto de calidad educativa disminuyó su protagonismo pero no su fuerza hegemónica. Del mismo modo, sin renunciar a la influencia de EFA, el gobierno de Calderón prefirió tomar como referencia la posición economicista del BM. Por ejemplo, el primer objetivo del Plan Sectorial 20072012, es "elevar la calidad de la educación para que los estudiantes mejoren su nivel de logro educativo, cuenten con medios para tener acceso a un mayor bienestar y contribuyan al desarrollo nacional” (MÉXICO, 2007, p. 11), por lo que la "evaluación será un instrumento fundamental en el análisis de la calidad, la relevancia y la pertinencia del diseño y la operación de las políticas públicas en materia de educación. La evaluación debe contemplarse desde tres dimensiones: como ejercicio de rendición de cuentas, 
como instrumento de difusión de resultados a padres de familia y como sustento del diseño de las políticas públicas" (MÉXICO, 2007, p. 11). El cambio es sutil pero claro: lo que mide la calidad ya no son los procesos de inclusión o el desarrollo de habilidades para aprender a lo largo de la vida, como propone EFA, sino el logro educativo medido con estándares nacionales -e internacionales- para poder rendir cuentas, es decir, la eficiencia y eficacia se imponen sobre la pertinencia y la relevancia, de acuerdo con la propuesta del BM. El instrumento predilecto por esta administración fue el ENLACE, prueba censal aplicada a la inmensa mayoría de los estudiantes del país y que terminó estableciendo un ranking de escuelas, impactó en el salario docente y en la distribución del gasto educativo (POY, 2014). Asimismo igual que el gobierno de Fox, la administración de Calderón sustentó sus principios de calidad educativa en pactos políticos con la cúpula sindical del SNTE, antidemocrática y corrupta, lo que implicó una aparente flexibilidad curricular y un control más férreo del magisterio. En este caso se llamó Alianza por la Calidad de la Educación (ACE), firmado en $2008 .^{8}$

Tener presente la imbricación de la propuesta de la UNESCO y del BM, nos permitirá comprender en buena medida las características de los planes de estudio de la asignatura de historia en la educación básica publicados en 2011 por las siguientes razones: a) la historia modifica su epistemología escolar creando el concepto de competencias para responder a los principios de pertinencia y relevancia y b) la historia diseña estándares curriculares (denominado aprendizajes esperados) específicos para responder a los criterios de eficacia y eficiencia. Esta transformación será posible gracias a las traducciones didácticas de la investigación en enseñanza de la historia que promueve la categoría de pensar históricamente desde ciertas visiones cientificistas y psicológicas.

\section{El lugar de la historia en la educación básica}

Los gobiernos panistas, además de basar su discurso educativo en el concepto de calidad, fueron extraordinariamente reformistas en el ámbito educativo. Podría decir que

\footnotetext{
${ }^{8}$ Es importante señalar que los pactos políticos de los gobiernos neoconservadores tuvieron fuerte oposición, sobre todo por la Coordinadora Nacional de Trabajadores de la Educación (CNTE).
} 
la educación en México vivió doce años en reforma permanente. ${ }^{9}$ Uno de los resultados es que la educación básica y obligatoria en México quedó conformada por tres niveles educativos: preescolar (3-6 años de edad), primaria (6-12 años de edad) y secundaria (11-15 años de edad) y que por primera vez se logró publicar un plan de estudios coherente entre los tres niveles. Basado en la idea de calidad educativa, el proyecto de articulación a la educación básica de 2011, trata de combinar explícitamente lo nacional, representado por el concepto de aprendizajes esperados (SECRETARÍA DE EDUCACIÓN PÚBLICA, 2011) y lo internacional, es decir, los estándares definidos por el Programme for International Student Assessment (PISA). Su concreción se encuentra en el perfil de egreso, que es manifestación explícita de lo que se quiere fabricar en la escuela. Al finalizar los estudios de educación básica, los estudiantes deben ser capaces de expresarse bien por escrito y oralmente; de argumentar y razonar para identificar problemas y proponer soluciones; de seleccionar y utilizar la información de diversas fuentes; de interpretar los procesos sociales, económicos, financieros y naturales; de ejercer sus derechos humanos y vivir democráticamente; de valorar la interculturalidad; de ser emprendedor; de cuidar su salud y al medio ambiente; de utilizar los recursos tecnológicos a su alcance y de apreciar la dimensión estética de la vida, entre otras cosas (SECRETARÍA DE EDUCACIÓN PÚBLICA, 2011, p. 32).

La asignatura escolar de historia tendrá la obligación de formar en consecuencia con esta idea de sujeto universal. Pero esta concepción de sujeto no es nueva, ni la historia propuesta en 2011 es tan innovadora. Ambas provienen de reformas educativas más amplias y que, aunque sea someramente, es necesario reseñar. A principios de los años noventa del siglo XX se iniciaron en casi toda América Latina una serie de reformas educativas que tuvieron como documento rector Educación y conocimiento: eje de la transformación productiva con equidad, publicado por la Comisión Económica para América Latina y el Caribe (CEPAL) y la UNESCO en 1992. Este fue parte importante de la expansión de la calidad educativa y de los senderos que se deberían transitar para alcanzarla: universalización de la educación básica, educación para el mercado laboral,

\footnotetext{
${ }^{9}$ Esta tendencia no se ha modificado. La nueva administración del PRI continúo con las reformas, ahora para consolidar la autonomía del INEE y para promover políticas que reducen los derechos laborales docentes.
} 
orientación tecnológica y pragmática a los contenidos del currículo, procesos de descentralización administrativa y financiera, reducción del gasto público en educación y una progresiva disminución de las responsabilidades del Estado en materia educativa (COMISIÓN ECONÓMICA PARA AMÉRICA LATINA Y EL CARIBE Y ORGANIZACIÓN DE NACIONES UNIDAS PARA LA EDUCACIÓN, 1992, p. 152). México, durante el gobierno de Carlos Salinas de Gortari, siguió prontamente el camino señalado. En 1992 firmó el Acuerdo Nacional para la Modernización de la Educación Básica (ANMEB), promulgó la Ley General de Educación, elevó a rango constitucional la obligatoriedad de la educación secundaria, reformó los planes curriculares de primaria y secundaria e inició un proceso de federalización educativa.

La historia sufrió modificaciones importantes en los programas de 1993 para primaria y secundaria, aunque no abandonó su función identitaria tradicional. La organización por áreas de conocimiento que funcionaba desde los años setenta, se desmembró, por lo que Ciencias Sociales quedó dividida en Historia, Geografía y Civismo. En historia se introdujo un enfoque de enseñanza basado en el constructivismo cognitivo español para definir las nociones de aprendizaje y se agregaron reinterpretaciones de la escuela historiográfica de los Annales francesa en su segunda generación, es decir, de base braudeliana (PLÁ, 2008). Los programas de 2011 mantienen principios de los planes de estudio de 1993, en especial el carácter formativo y la visión de la historia como conocimiento en constante construcción (SECRETARÍA DE EDUCACIÓN PÚBLICA, 2009, p. 20), pero sobre todo perfecciona el enfoque procedimental de la historia hasta ubicarlo jerárquicamente por encima del conocimiento del pasado. Es decir, la pertinencia y la relevancia de la historia en el currículo se basan en sus componentes técnicos y no interpretativos. Esta idea se concretó en 2006 con la reforma a la educación secundaria, cuando se inventaron didácticamente las competencias disciplinares del saber histórico: comprensión del tiempo y espacio histórico, manejo de información histórica y formación de una conciencia histórica para la convivencia democrática e intercultural (SECRETARÍA DE EDUCACIÓN PÚBLICA, 2006, p. 9).

La Reforma Integral a la Educación Básica (RIEB) iniciada en 2009 y terminada en 2011 consiguió un plan de estudios sólido y coherente, lo que facilita, por lo menos en el 
ámbito prescriptivo, establecer un continuum entre tres niveles educativos (preescolar, primaria y secundaria). La historia se imparte en la actualidad en cuatro grados de primaria y dos de secundaria. En primaria, el tercer grado se concentra en historia y geografía de la entidad, lo que implica que los contenidos son seleccionados por cada estado de la federación; en cuarto y quinto se trabaja historia de México y en sexto historia de México y el mundo (hasta el siglo XVI). En secundaria se estudia en segundo grado, después de un año de ausencia de la asignatura, desde el siglo XVI hasta la actualidad y en el último año de educación obligatoria, se retoma la historia de México desde la etapa virreinal a la fecha. 2011 no modifica esta estructura ni las competencias, pero reduce exitosamente las temáticas del programa y se centra en aspectos procedimentales del aprendizaje de la historia. Estos veinte años de reforma neoliberal han visto como los programas de historia sufrieron una significativa metamorfosis de su epistemología, pasando de una historia memorística y enciclopédica a una historia práctica, metodológica, "relevante" y "pertinente" para la vida cotidiana y el futuro laboral de los estudiantes.

Los contenidos históricos propuestos en diferentes momentos de las reformas panistas entre 2000 y 2012 no estuvieron exentos de oposición. La más relevante sucedió en 2004, cuando se hizo público el mapa curricular preliminar para la educación secundaria. En él la historia se enseñaba solo en tercer grado con una carga horaria de 8 horas por semana y con contenidos de historia de México y del Mundo desde finales del siglo XV a la actualidad. La polémica se centró en los contenidos desde dos dimensiones. Una fue que la opinión pública consideraba necesario separar la historia de México de la historia universal porque se perdía la especificidad histórica de la nación y la identidad mexicana. La segunda fue consecuencia de un importante proceso de exclusión. Con el argumento que en primaria se trabajan los pueblos mesoamericanos y que ya no era necesario estudiarlos en secundaria, se decidió iniciar el curso de historia con los viajes de descubrimiento y la llegada de los españoles a América. Para la inmensa mayoría de la población y en especial para los historiadores (ARREDONDO, 2005), el programa retomaba visiones hispanistas y conservadoras de la historia nacional, en el que el origen de lo mexicano se encontraba en la conquista de México y Nueva España y no en 
Mesoamérica. En otros espacios he argumentado que la intención de excluir a los pueblos indígenas, además de su tono hispanista, tenía la finalidad de construir un metarrelato nacional basado en los procesos de globalización y en la recuperación de los organismos financieros internacionales como sujetos históricos destacados (PLÁ, 2012). La exclusión de la historia indígena y el aumento de la historia virreinal son claramente un intento neoconservador de reescribir la historia que se enseña. La inclusión de los organismos financieros internacionales como sujetos históricos relevantes es el lado neoliberal de los panistas.

Independientemente de las polémicas generadas por el programa preliminar y que terminaram modificándolo al grado de incluir el estudio de los pueblos prehispánicos en tercero de secundaria y dividir en dos cursos (de México y Universal) los contenidos de historia, considero importante señalar que el enfoque por competencias y la organización programática por aprendizajes esperados no fueron cuestionados. Tampoco lo fueron en 2009 cuando un grupo de profesores michoacanos decidió promover la "verdadera" historia, pues el programa para educación primaria omitía acontecimientos importantes, como los Acuerdos de Bucareli de 1923, donde el gobierno mexicano pactó con las trasnacionales petroleras que no haría retroactivo el artículo 27 constitucional, artículo que exigía la expropiación del petróleo. Las razones de esta falta de cuestionamiento a mi parecer son que el discurso de calidad educativa se ha naturalizado a tal grado que su enfoque procedimental ya no es cuestionado, a pesar que en la enseñanza de la historia la importancia social de los contenidos sigue siendo para la mayoría lo más relevante. La segunda es que la didáctica de la historia y ciertas interpretaciones del pensar históricamente, se han encargado de eliminar la importancia del pasado para convertir a la enseñanza en un mero desarrollo de habilidad. Ahí se encuentra uno de los vínculos más estrechos entre la nueva didáctica de la historia y el discurso de calidad educativa.

\section{Competencias y aprendizajes de la historia}

Los programas de estudio de todas las asignaturas de educación básica deben de responder al perfil de egreso del estudiante a través de tres elementos centrales: el 
desarrollo de competencias, los estándares curriculares y los aprendizajes esperados. Cada uno de ellos tiene su propio sentido, pero todos deben ser mensurables. La SECRETARÍA DE EDUCACIÓN PÚBLICA define el quinto principio pedagógico rector de los programas para la articulación de la educación básica de 2011 como:

Una competencia ${ }^{10}$ es la capacidad de responder a diferentes situaciones, e implica un saber hacer (habilidades) con saber (conocimiento), así como la valoración de las consecuencias de ese hacer (valores y actitudes).

Los Estándares Curriculares son descriptores de logro y definen aquello que los alumnos demostrarán al concluir un periodo escolar [...]. Los Estándares Curriculares son equiparables con estándares internacionales $y$, en conjunto con los aprendizajes esperados, constituyen referentes para evaluaciones nacionales e internacionales que sirvan para conocer el avance de los estudiantes durante su tránsito por la Educación Básica $[\ldots]$.

Los aprendizajes esperados son indicadores de logro que, en términos de la temporalidad establecida en los programas de estudio, definen lo que se espera de cada alumno en términos de saber, saber hacer y saber ser $[\ldots]$.

Las competencias, los Estándares Curriculares y los aprendizajes esperados proveerán a los estudiantes de las herramientas necesarias para la aplicación eficiente de todas las formas de conocimientos adquiridos, con la intención de que respondan a las demandas actuales y en diferentes contextos. (SECRETARÍA DE EDUCACIÓN PÚBLICA, 2011, p. 22)

En estos pocos párrafos se concentra el proceso de imbricación de la propuesta de calidad educativa del BM y de la UNESCO. La competencia se convierte en el objetivo a lograr, los estándares curriculares son criterios de logro que permiten establecer parámetros comparativos entre evaluaciones nacionales e internacionales y, finalmente, los aprendizajes esperados son una especie de indicadores que se deben cubrir para alcanzar los estándares y de ahí aprender las competencias. La imbricación entre ambos discursos de calidad consiste en conjuntar la idea de necesidades básicas de aprendizaje con formas de medición eficiente de los procesos de enseñanza y aprendizaje y de la eficacia de la política educativa a partir de resultados. Todos juntos serán fundamentales para dar las directrices a las nuevas configuraciones de los saberes escolares.

\footnotetext{
${ }^{10}$ Negritas en el original
} 
¿Cómo responde la historia a esto? Definiendo propósitos, inventando competencias y señalando aprendizajes esperados para articular lo que se entiende por pensar históricamente. La historia en educación básica en México tiene como propósitos que los alumnos desarrollen nociones espaciales y temporales para la comprensión de procesos y hechos históricos y habilidades para el manejo de información histórica; reconozcan que son parte de la historia, con identidad nacional y mundial; y participen de manera informada y democrática en su sociedad (SECRETARÍA DE EDUCACIÓN PÚBLICA, $2011^{\mathrm{a}}$, p. 13). Por su parte, las competencias son tres:

Comprensión del tiempo y del espacio históricos. ${ }^{11}$ Favorece que los alumnos apliquen sus conocimientos sobre el pasado y establezcan relaciones entre las acciones humanas, en un tiempo y un espacio determinados, para que comprendan el contexto en el cual ocurrió un acontecimiento o proceso histórico. Esta competencia implica el desarrollo de las nociones de tiempo y de espacio históricos, el desarrollo de habilidades para comprender, comparar y analizar hechos, así como reconocer el legado del pasado y contar con una actitud crítica sobre el presente y devenir de la humanidad.

Manejo de información histórica. El desarrollo de esta competencia permite movilizar conocimientos, habilidades y actitudes para seleccionar, analizar y evaluar críticamente fuentes de información, así como expresar puntos de vista fundamentados sobre el pasado.

Formación de una conciencia histórica para la convivencia. Los alumnos, mediante esta competencia, desarrollan conocimientos, habilidades y actitudes para comprender cómo las acciones, los valores y las decisiones del pasado impactan en el presente y futuro de las sociedades y de la naturaleza. Asimismo, fomenta el aprecio por la diversidad del legado cultural, además del reconocimiento de los lazos que permiten a los alumnos sentirse parte de su comunidad, de su país y del mundo (SECRETARÍA DE EDUCACIÓN PÚBLICA, 2011ª p. 23-24).

Parte sustancial del discurso de calidad educativa consiste en establecer una supuesta proximidad entre el conocimiento científico y el conocimiento escolar. Mientras más próximos estemos en la escuela de las formas de pensar y producir la ciencia, más relevantes serán los aprendizajes. Los niveles de desempeño en ciencias de los exámenes PISA de la OCDE son ejemplo de esta aparente proximidad. Pero la cercanía es ilusoria y se puede ver con facilidad en las competencias diseñadas para la enseñanza de la historia a imagen y semejanza de lo que se considera pensamiento histórico.

\footnotetext{
${ }^{11}$ Negritas en el original
} 
El tiempo y el espacio históricos como noción de aprendizaje son creaciones didácticas y psicológicas, pero no historiográficas. El objeto de investigación creado por los historiadores no es el tiempo o en su caso el espacio, sino el ser humano en sociedad en un espacio y en un tiempo determinado. Sin embargo, en la necesidad -primero de los psicólogos constructivistas de buscar la especificidad del pensamiento histórico en un sujeto cognoscente universal y después de las políticas educativas para medir la calidad educativa a partir de resultados- por encontrar un esquema válido de pensar el pasado, determinaron que el tiempo y el espacio históricos son competencias cognitivas. Aunque comienzan a ganar lugar en el currículum iberoamericano a finales de los ochenta y con gran fuerza en los noventa (CARRETERO,ASENSIO y POZO, 1989; PAGÉS, 2002) el tiempo y el espacio histórico como preocupación de la psicología pueden rastrearse hasta las primeras investigaciones de Gustav Jahoda en los años sesenta e incluso antes (CARRETERO, ASENSIO y POZO, 1989; WINEBURG, 1996). Sustentadas en estas investigaciones, las propuestas para la enseñanza de la historia atraen desde la psicología la noción de sujeto cognoscente universal y lo convierten en objeto mensurable y por tanto objeto de enseñanza, arguyendo proximidad con el saber disciplinar.

La segunda competencia es de nuevo un velo que cubre al sujeto cognoscente universal con lo disciplinar. Cuando se sostiene que la historia debe enseñar habilidades específicas del saber histórico para analizar y seleccionar críticamente diferentes fuentes de información, en realidad se hace mención a habilidades concebidas como genéricas y no exclusivas del quehacer de los historiadores. El perfil de egreso de la educación básica nos presenta otro ejemplo de estas competencias genéricas, pues se pretende que el estudiante obtenga la capacidad de seleccionar, analizar, evaluar y compartir información proveniente de diversas fuentes y sea capaz de utilizar el lenguaje oral y escrito de manera fluida (SECRETARÍA DE EDUCACIÓN PÚBLICA, 2011, p. 32). Es, nuevamente, la construcción de una forma de pensar aparentemente universal y expandida con fuerza desde la proclamación de EFA en 1990. Por ejemplo, un significado similar y renovado puede verse en la definición de código de la modernidad defendido por la CEPAL y la UNESCO en 1992: es "el conjunto de conocimientos y destrezas necesarios para participar en la vida pública y desenvolverse productivamente en la sociedad moderna”. 
El código de la modernidad define sus capacidades como las requeridas para el manejo de las operaciones aritméticas básicas; la lectura y comprensión de un texto escrito; la comunicación escrita; la observación, descripción y análisis crítico del entorno; la recepción en interpretación de los mensajes de los medios de comunicación modernos; y la participación en el diseño y la ejecución de trabajos en grupo [y por tanto] constituye a su vez el patrón de medida para evaluar el diseño de los currículos y la eficacia de las metodologías aplicadas en el proceso pedagógico (COMISIÓN ECONÓMICA PARA AMÉRICA LATINA Y EL CARIBE Y ORGANIZACIÓN DE NACIONES UNIDAS PARA LA EDUCACIÓN, 1992, p. 157).

La tercera competencia no es un intento de proximidad con la ciencia histórica. Formar en el respeto a la interculturalidad no tiene nada que ver con el saber disciplinar, sino con el código disciplinar de la historia (CUESTA, 1997), ${ }^{12}$ que en los últimos veinte años pasó de una identidad nacional monolítica a la idea de un México pluricultural. La tradicional función identitaria del uso público de la historia y sus manifestaciones dentro de los sistemas educativos perduran en las propuestas para la enseñanza de la historia de 2011 (PLÁ, 2009; p. 323-324). Esto no implica que en el momento de diseñar los contenidos históricos, la pluriculturalidad sea opacada por una visión conservadora e hispanista de la historia mexicana.

Por último, la historia al ser un conocimiento social que no es evaluado internacionalmente y por tanto no existen estándares con los cuales compararla, carece de estándares curriculares para su aprendizaje y evaluación. Lo que si posee es una larga lista, sólo en secundaria, de cuarenta aprendizajes esperados que van desde valorar los aportes de la antigüedad hasta reconocer las características y la importancia de la globalización en la actualidad. Estos han sido objeto de evaluaciones nacionales y sin duda la más importante fue en 2010, cuando los resultados que arrojó ENLACE sobre el conocimiento histórico y el desempeño de los estudiantes no solo fue muy deficiente, sino que el diseño de la prueba fue en contra del enfoque de enseñanza porpuesto por la SECRETARÍA DE EDUCACIÓN PÚBLICA, pues retomó más aspectos de la historia memorística que de la historia formativa. (PLÁ, 2011; CAMARGO, 2012) Lo relevante, a mi

\footnotetext{
${ }^{12}$ Cuesta define al código disciplinar de la historia como un saber que tiene una racionalidad distinta de la historiografía profesional y que se ha construido históricamente. Es un conjunto de valores, rutinas, suposiciones y valores que fundamentan la inclusión de la historia dentro del currículum de educación básica.
} 
parecer, es por un lado la similitud entre la pedagogía por objetivos de Benjamín Bloom y la definición de aprendizajes esperados en la actualidad y por otro la ubicación del conocimiento histórico como conocimiento inferior frente a las habilidades comunicativas, el pensamiento matemático y las ciencias naturales. Su supervivencia en el currículo entonces se debe quizá más a la tercera competencia y la representación social que se tiene sobre la función social de la historia escolar, que a su potencialidad para desarrollar un pensamiento científico.

Pero la historia en el currículo no se transformó sola o por un acto de voluntad indefinido. Hubo personas, especialistas en el proceso de transposición didáctica, ya no del saber sabio al saber enseñado, sino de un sujeto cognoscente universal, al saber escolar a través de la categoría de pensar históricamente. Es necesario, entonces explicar la función de diferentes actores en este proceso.

\section{Didáctica de la historia y pensar históricamente}

Pensar históricamente es una categoría muy estudiada en la enseñanza de la historia. $Y$ aunque existen diferentes posicionamientos teóricos, por ejemplo la mirada sociocultural de Keith Barton y Linda Levstik (2004) no es igual a las propuestas más disciplinares y cognitivas de Mario Carretero (2011) o Samuel Wineburg (2001), hay relativo consenso. Mi intención no es describir las diferencias en las teorías del pensamiento histórico, sino indagar sobre las procedencias teóricas que influyeron en los responsables de la didáctica de la historia de la SECRETARÍA DE EDUCACIÓN PÚBLICA y su impacto en la producción política de la historia como saber escolar. Por eso, en este apartado me limito a resumir qué se entiende por pensar históricamente para los autores citados por los didactas mexicanos y posteriormente describir los diferentes grupos de poder que definieron contenidos y enfoques de enseñanza para la educación básica en México. Esto implica necesariamente ser consciente de que la mayoría de los investigadores citados no son responsables directos de su impacto en los programas de estudio, con excepción quizá de Mario Carretero y su trabajo de asesor de los programas preliminares de secundaria de 2004. 
Este último autor también puede servirnos de guía para definir pensar históricamente y su impacto en la didáctica mexicana. Mario Carretero publica en 2011 un texto dedicado a las investigaciones cognitivas sobre pensar históricamente. Este texto es parte de un documento publicado por la SECRETARÍA DE EDUCACIÓN PÚBLICA titulado Enseñanza y aprendizaje de la Historia en Educación Básica y que tiene la función de homogenizar la interpretación docente sobre diversas temáticas relacionadas con la enseñanza de la historia. En un muy apretado resumen y por tanto con riesgo de simplificar, puedo decir que Carretero afirma que el pensar históricamente en cuanto habilidad cognitiva ha podido ser definido gracias a las potencialidades del método de comparación entre expertos y novatos, es decir, que el pensar históricamente hace referencia en última instancia a la producción de conocimiento histórico por parte de los historiadores. Sus componentes básicos son los conceptos de primer orden (acontecimientos, conceptos, instituciones históricas, etc.) y segundo orden (causalidad, temporalidad, etc.); la dimensión temporal de toda interpretación histórica; el planteamiento y la resolución de problemas históricos; el uso de fuentes primarias para producir el conocimiento histórico y su carácter narrativo (CARRETERO, 2011). Esta posición me permite ver por lo menos dos aspectos: por un lado los componentes del pensar históricamente no se alejan mucho del sujeto cognoscente universal definido para una educación de calidad (pensamiento complejo, uso de información, resolución de problemas). Por otro, el papel que juega la psicología al sustentar "científicamente" la existencia del sujeto universal. La didáctica de la historia en México será la responsable de conjuntar psicología, historia, docencia y calidad educativa.

La didáctica de la historia propuesta por la SECRETARÍA DE EDUCACIÓN PÚBLICA no es libre y está determinada, al igual que otras didácticas específicas, por las exigencias del marco curricular común y por las capacidades de decisión de distintos grupos de poder. En las reformas de 2011 podemos observar la presencia de por lo menos dos equipos de especialistas en didáctica de la historia. Por un lado, se encuentra el sector comandado por Laura Lima Muñiz, quien dominó los programas de historia, los materiales didácticos y todo el proceso de sedimentación de la propuesta hegemónica desde los primeros años de la década de los noventa, hasta los propios planes de estudio 
de 2011. Parte constitutiva de su equipo es Felipe Bonilla Castillo. Por otro lado, se encuentra el grupo que determinó los contenidos y los enfoques de educación histórica para algunas asignaturas del plan de estudios 2012 de la Licenciatura en Educación Primaria. En él se encuentran entre otros Belinda Arteaga y su hijo Siddhartha Camargo. Ambas agrupaciones están lideradas por académicas de la Universidad Pedagógica Nacional (UPN), institución de educación superior fundada en 1978 y que tuvo entre sus objetivos primigenios ofrecer servicios especializados a la SECRETARÍA DE EDUCACIÓN PÚBLICA.

No se pueden entender los procesos de transformación de la historia en los programas de estudio para la educación básica en México y de la nueva didáctica de la historia esgrimida por la SECRETARÍA DE EDUCACIÓN PÚBLICA sin tomar en cuenta al primer grupo y en especial a Laura Lima. Esta académica de la UPN ocupó cargos importantes dentro de la administración federal. Primero, como Directora del Área de Ciencias Sociales de la Dirección General de Materiales y Métodos Educativos de la SECRETARÍA DE EDUCACIÓN PÚBLICA fue parte del grupo que diseñó los contenidos y los materiales de apoyo de las asignaturas de Historia de la Licenciatura de Educación Primaria para las escuelas normales en 1999, revisó el cumplimiento de los enfoques en los libros de texto para primaria de la asignatura de historia y geografía en diferentes entidades y posteriormente, como Directora del Área de Seguimiento y Evaluación Curricular de la Dirección General de Desarrollo Curricular de la Subsecretaría de Educación Básica estuvo a cargo del enfoque de enseñanza para la asignatura de historia y del comité interinstitucional de historia que definió los contenidos en la reforma a la educación secundaria de 2006. Ya lejos del cargos públicos siguió asesorando a la SECRETARÍA DE EDUCACIÓN PÚBLICA y difundiendo su pensamiento (LIMA, BONILLA y ARISTA, 2010). En resumen, Laura Lima es una de las principales ideólogas y académicas en la producción y difusión del nuevo enfoque para la enseñanza de la historia desde las reformas de 1992 hasta la actualidad.

Cuando analizamos el pensamiento didáctico de Lima es necesario tener presente que su producción es directamente política, de intervención y de gran impacto y no teórica o de investigación. Sus productos son los enfoques de enseñanza, la selección de 
contenidos y las concepciones de historia que subyacen en los programas de estudio para todas las escuelas primarias y secundarias del país. Por tanto, su loci de enunciación es el poder. Asimismo, al estar su obra enmarcada en las políticas curriculares oficiales, nunca explicita sus procedencias teóricas o los referentes de investigación en los que sustenta su propuesta. Sin embargo, a pesar de estas dificultades analíticas, los planes de estudio y las pocas entrevistas que ha ofrecido (LIMA, 2005) muestran una fuerte influencia de la didáctica de la historia española de finales de los ochenta y principios de los noventa del siglo pasado y en especial de Mario Carretero (1989), ${ }^{13}$ así como de Victoria Lerner (1993, 1995), Mireya Lamoneda (1990, 1998) ${ }^{14}$ y Andrea Sánchez Quintanar (2004). Tanto para la investigación española como para las investigadoras mexicanas, el currículo de historia debería estar diseñado en torno de las nociones de tiempo y espacio históricos. El resultado más concreto de esto es sin lugar a dudas la invención contemporánea del tiempo y espacio históricos como competencias (SECRETARÍA DE EDUCACIÓN PÚBLICA, 2011). Otras características son la influencia del constructivismo cognitivo y su reinterpretación de Jean Piaget, la influencia de la escuela historiográfica francesa de los Annales y en especial de Fernand Braudel (PLÁ, 2008) y el uso de fuentes primarias para la enseñanza de la historia en el aula.

Lima y Bonilla no sostienen con claridad una definición de pensar históricamente. Lo que se puede distinguir entre líneas en sus textos y de los documentos oficiales que produjeron es que pensar históricamente es básicamente concebir la dimensión temporal de los procesos sociales. Por ejemplo la enseñanza basada en datos y fechas de corte memorístico no enseñaba a "pensar históricamente, pues no se consideraba importante la ubicación y comprensión temporal y espacial de los hechos y procesos históricos, por

\footnotetext{
${ }^{13}$ Laura Lima cursó el diplomado Enseñanza de las Ciencias Sociales: Construcción del conocimiento y actualización disciplinar. FLACSO-Argentina donde Mario Carretero es profesor. Además, Carretero fue asesor de Lima en 2003, durante la primera etapa de la reforma a la educación secundaria y que concluyó en 2006.

${ }^{14}$ Mireya Lamoneda fue protagonista en la concepción de tiempo histórico que predomina hoy en los currículos oficiales, sobre todo en su manifestación gráfica denominada línea del tiempo. Lamoneda fue coordinadora del equipo de evaluación y dictaminación de los libros de texto para educación secundaria escritos a partir de la reforma a los planes de estudio de 1993 hasta su fallecimiento en 2003, es decir, regulaba el cumplimiento de las normas educativas para definir las formas legítimas de enseñar la historia.
} 
supuesto ni a establecer relaciones causales con otros acontecimientos nacionales o mundiales" (LIMA, BONILLA y ARISTA, 2010, p. 4). En otro texto cordinado por Bonilla se define de manera más amplia: "Pensar históricamente implica reconocer que todos los seres humanos dependemos unos de otros y que nuestro actuar presente y futuro está estrechamente relacionado con el pasado. [...]. Por tanto, el pensamiento histórico implica un largo proceso de aprendizaje que propicia que los alumnos, durante la Educación Básica y de manera gradual, tomen conciencia del tiempo, de la importancia de la participación de varios actores sociales, y del espacio en que se producen los hechos y procesos históricos" (SECRETARÍA DE EDUCACIÓN PÚBLICA, 2011ª p.16). Esta acepción será central para definir los aprendizajes esperados, pues a lo largo del programa de historia de primaria y secundaria se establecen la ubicación temporal y espacial de los contenidos trabajados como primer objetivo. Asimismo, suplanta a partir de ciertas nociones de pensar históricamente al conocimiento del pasado por el desarrollo de las habilidades cognitivas como objetivo central de enseñanza.

El segundo grupo llegó después y no ha tenido la misma fuerza de largo plazo del primero. Sin embargo, esto no resta el gran impacto de su trabajo, pues implica un cambio curricular a la formación de todos los docentes de educación primaria que se forman en las escuelas normales mexicanas. Parte importante de sus logros se debieron a dos aspectos: el primero fue que Siddhartha Camargo fue subdirector de Enfoques y Contenidos de la Educación Normal de la Dirección General de Educación Superior para Profesionales de la Educación (DGESPE) de la SECRETARÍA DE EDUCACIÓN PÚBLICA, lo que les permitió desarrollar un proyecto de largo aliento denominado Comunidad Normalista para la Educación Histórica que facilitó la discusión y el diseño curricular con profesores de las escuelas normales de toda la república. La segunda, es que las reuniones colegiadas estuvieron sustentadas en investigaciones contemporáneas en enseñanza de la historia, sobre todo la producida por Peter Seixas y Carla Peck (2008) y Sam Wineburg (2001), además de retomar los estudios de Lee Shulman (2005) para apoyar la formación docente y de los trabajos de Andrea Sánchez Quintanar (2004) y su figura de historiador-docente. Además de las propias investigaciones y textos de Arteaga y Camargo, entre los que sobresale por mucho el Estudio de cultura y conciencia histórica 
en las Escuelas Normales de 2011, el proyecto de Educación Histórica derivó en la creación de archivos históricos en varias escuelas normales. Sin lugar a dudas, el trabajo de Arteaga y Camargo es, desde la política educativa para la enseñanza de la historia, el más innovador y productivo que ha tenido México en mucho tiempo.

Aunque el trabajo de Lima considera importante las características de la disciplina histórica como componente para su enseñanza, como lo demuestra la competencia de manejo de información histórica (SECRETARÍA DE EDUCACIÓN PÚBLICA, 2011), no alcanza los niveles de complejidad y radicalismo de Arteaga y Camargo. La propuesta de estos últimos, denominada educación histórica, es un esfuerzo deliberado por llevar al aula el pensamiento histórico lo más parecido posible al pensamiento experto, es decir, la enseñanza de la historia no debe implicar una discusión de contenidos sino saber cómo formar a los estudiantes en la manera de investigar de los historiadores, es decir, pensar históricamente como un tipo particular de conocimiento (ARTEAGA y CAMARGO, 2010). La educación histórica implica enseñar una forma de investigar. Esto implica la superación de dos formas de educación histórica que le antecedieron: la narrativa nacional y la función de la historia para comprender el presente. Por tanto, para estos autores, lo más importante de la didáctica de la historia es el uso de fuentes primarias y el aprendizaje de los conceptos de segundo orden -siguiendo a Peter Lee y Rosalyn Ashby (2000)-, que se utilizan para la interpretación del pasado, es decir, el tiempo histórico, la causalidad, cambio y permeancia y empatía entre otros. Tan radical es la posición de Arteaga y Camargo que el problema del contenido es casi irrelevante y toda intromisión política o cultural a esta lógica es una interferencia del objetivo central de la educación histórica: defender a la historia como ciencia y por tanto su valor para la fabricación del sujeto cognitivo universal. Esta interpretación es simbiótica con la calidad educativa pues crea la sensación de proximidad entre ciencia, aprendizaje y evaluación.

Las diferencias entre estos dos grupos son menores que las semejanzas. El proyecto de Arteaga y Camargo está mucho mejor fundamentado en la investigación en enseñanza de la historia que el de Lima. El primero tiene un trabajo colegiado con docentes de la escuela normal que el segundo no tiene. La propuesta de educación histórica utiliza con mayor claridad la epistemología del conocimiento histórico y la teoría 
de la historia, mientras que los trabajos de Lima siguen anclados en la segunda generación de los Annales. Por último, el trabajo de Lima está mucho más supeditado a las directrices generales de los planes y programas de estudio para la educación básica, mientras que Arteaga y Camargo trabajan con menos ataduras. Pero la lista de diferencias no aumenta mucho más. En cambio las similitudes son numerosas y que, por motivos de espacio, trataré se sintetizar lo más posible. Ambos grupos consideran que la disciplina histórica es la fuente primigenia del conocimiento histórico escolar; que la metodología de la historia, en especial el uso de fuentes, debe ser el núcleo de los quehaceres didácticos; los dos establecen la relación jerárquica en la que el desarrollo cognitivo se encuentra por encima del conocimiento del pasado; los dos al concebir esquemas de pensamiento o conceptos de segundo orden o metaconceptos, terminan produciendo una historia en apariencia ahistórica, es decir, metahistórica; y los dos construyen modelos de pensamiento histórico fácilmente comunicables y evaluables.

La didáctica de la historia ha cumplido un papel central en la conversión de un saber histórico escolar básicamente narrativo, memorístico y nacionalista a un saber escolar centrado en las formas de producción del conocimiento profesional o experto. Esta transformación ha generado propuestas más complejas y en muchos aspectos más incluyentes que las formas tradicionales de enseñanza. Sin embargo, esto no implica que no genere nuevas formas de exclusión. La primera de ellas, basada en la idea de sujeto cognoscente universal de la calidad educativa se centra en la negación de cualquier forma alternativa de pensar el pasado que no sea lo que se define como pensamiento histórico legitimo. La segunda y no por eso menos importante, es la exclusión didáctica del problema del contenido histórico -situación que no es aceptada por los profesores, incluso por algunos historiadores y por la sociedad en general, como su pudo ver en los movimientos de oposición a las reformas a la educación secundaria en 2004-, pues se considera que este problema es político y desvía la atención de lo "verdaderamente" importante, es decir el desarrollo de habilidades cognitivas evaluables y que respondan a los discursos de calidad educativa. Lo que no ve esta posición tan cientificista -y psicológica- de la historia es que niega la propia dimensión política de la producción del 
conocimiento histórico o como decía con cierta ironía un ilustre historiador mexicano hace ya más de sesenta años: la

elevación de la historia a la 'dignidad de la ciencia', no es sino el refinamiento técnico de la investigación que da lugar a una renovada metodología, pero que, sin embargo, [...] está toda ella animada e inspirada por los propósitos pragmáticos e interesados del historiador. Lo que acontece es que la nueva metodología [... oculta cada vez más esos propósitos (O'GORMAN, 1947, p. 41-42).

Trasladado a la pedagogía contemporánea, podemos decir que la calidad educativa y la didáctica de la historia basada en el pensamiento histórico como método ocultan su propia intencionalidad política bajo la idea de método y de ciencia.

Por último se encuentra la relación entre pensar históricamente y evaluación. La necesidad de crear parámetros curriculares del aprendizaje de la historia con base ciertas definiciones cientificistas y cognitivas de pensar históricamente que concuerden con la relevancia y la eficacia medida en el logro académico a gran escala, termina por justificar científicamente el carácter profundamente excluyente o de injusticia cognitiva (SOUSA SANTOS, 2010) ${ }^{15}$ del sujeto cognitivo universal difundido por la EFA y el BM. Sostengo lo anterior porque al concentrarnos en habilidades cognitivas surgidas desde una racionalidad occidental (los historiadores) desviamos la atención del problema cultural que implica la imposición de unos parámetros curriculares para evaluar las formas culturales de pensar el pasado. En otras palabras, damos por hecho la existencia de una verdad universal que llamamos calidad educativa.

${ }^{15}$ La injusticia cognitiva para Boaventura Sousa Santos es: "Ya que el conocimiento científico no está socialmente distribuido de manera proporcionada, las intervenciones en el mundo real que favorece tienden a ser aquellas que atienden a los grupos sociales que tienen acceso al conocimiento científico. La injusticia social se basa en la injusticia cognitiva. Sin embargo, la lucha por la justicia cognitiva no tendrá éxito si se sustenta únicamente en la idea de una distribución más equilibrada del conocimiento científico. Aparte del hecho de que esta forma de distribución es imposible en las condiciones del capitalismo global, este conocimiento tiene límites intrínsecos con relación a los tipos de intervención que se pueden alcanzar en el mundo real. Estos límites son el resultado de la ignorancia científica y de una incapacidad para reconocer formas alternativas de conocimiento de interconectar con ellas en términos de igualdad. En la ecología de saberes, forjar credibilidad para el conocimiento no científico no supone desacreditar el conocimiento científico. Simplemente implica su utilización contrahegemónica. Consiste, por una parte, en explorar prácticas científicas alternativas que se han hecho visibles a través de las epistemologías plurales de las prácticas científicas y, por otra, en promover la interdependencia entre los conocimientos científicos y no científicos" (SOUSA SANTOS, 2010, p. 51) 


\section{Calidad educativa y didáctica de la historia}

Las reflexiones anteriores arrojan una serie de temas o problemáticas diversas sobre la relación entre propuestas de calidad educativa y didáctica de la historia que he tratado de sintetizar en cinco puntos:

1) La enseñanza de la historia en los últimos veinte años en México ha sufrido transformaciones en varios aspectos muy positivos. Por un lado se ha tratado de evitar una historia memorística y enciclopédica para enseñar una historia más formativa y reflexiva. También se han reducido los contenidos históricos y se han propuestos ámbitos que sobrepasan lo político o lo económico, dando entrada por ejemplo a la historia cultural. En cierta medida es una propuesta más incluyente que la anterior. Sin embargo, el resultado todavía no alcanza la historia plural que requiere un país con la diversidad cultural de México y termina por generar nuevas exclusiones.

2) Durante los gobiernos conservadores del PAN entre 2000 y 2012 se notó un impulso exacerbado por la calidad educativa que completó la transformación de las epistemologías de los saberes escolares iniciada en la última década del siglo XX. Las principales mutaciones se dieron para responder a los criterios de eficacia y de relevancia. El primero se hizo a través de estándares curriculares que respondiera a las exigencias a la evaluación masiva, nacional e internacional, para medir la eficacia con base en los logros de aprendizaje. La relevancia se desarrolla a partir de la formación en habilidades cognitivas que se consideran universales y que han sido condensadas en México y otras partes del mundo en el término de competencias.

3) La historia en la escuela no queda exenta de estas modificaciones y crea estándares y competencias que le permitan responder a los criterios de relevancia y eficacia. Las competencias se denominan tiempo y espacio histórico, manejo de información histórica y conciencia histórica. Cada una de ellas se desglosa en una serie de habilidades que se sustentan teóricamente en los resultados de investigaciones sobre las características del pensamiento histórico o pensamiento experto. Los estándares de aprendizaje se llaman aprendizajes esperados y son un listado de objetivos cognitivos y 
de conocimientos históricos que el alumno debe aprender a lo largo de la educación básica. Entre ambas apoyan la producción del sujeto cognoscente universal.

4) La didáctica de la historia en México ha sido parte fundamental del proceso de transformación de la epistemología del conocimiento histórico escolar. Su función básica ha sido sostener -con base en principios científicos- la idea de una sola forma legítima de pensar el pasado dentro de la escuela. Esto implica desviar la atención no sólo del problema del contenido histórico, sino de la propia racionalidad de calidad educativa como imposición cultural. En este sentido las interpretaciones cientificistas del pensar históricamente han sido utilizadas por la didáctica de la historia en México para desviar la atención del problema del contenido histórico y naturalizar la idea de calidad educativa.

5) A pesar de que la investigación científica educativa y el discurso de calidad educativa se encuentre naturalizado en la jerga pedagógica mexicana es muy probable que maestros y diversos sectores de la población sigan luchando contra la injusticia cognitiva que implica no reconocer su derecho a pensar la historia con diferentes estructuras a las impuestas por los currículos neoconservadores. Para muchos de ellos, no se trata de negar el pensamiento científico, sino proponer una interdependencia entre otras distintas formas de temporalidad, de explicaciones históricas o de narrativas sobre los pasados. Sin embargo, todo parece indicar que la OCDE, la UNESCO y la calidad educativa mexicana, mantengan al conocimiento histórico en una posición jerárquicamente inferior a pesar de todos los esfuerzos de ciertos especialistas por volver al pensar históricamente en pensamiento científico. Esto sucederá porque una y otra vez, la interdependencia de conocimientos sobre el pasado les demuestra el castillo de naipes sobre el que se sostiene su idea de sujeto cognoscente universal.

\section{Referencias}

APPLE, Michael W. Educar "como Dios manda": mercados, niveles, religión y desigualdad. Barcelona: Paidós, 2002.

ARREDONDE, Adelina. Reforma integral a la educación secundaria en historia. Inventio. México. n. 2, p. 29-45, 2005. 
ARTEAGA, Belinda;CAMARGO, Siddharta. Educación histórica:una propuesta para el desarrollo del pensamiento histórico en los estudiantes de la licenciatura en educación preescolar y primaria. México: Dgespe, 2010. Disponible en

http://www.dgespe.sep.gob.mx/public/comunidades/historia/recursos/educacion_historic a_19_de_agosto_2012.pdf. Consultado:25/02/2014

BARRETT, Angeline M.; CHAWLA-DUGGAN, Rita; LOWE, John; UKPO, Eugenia. The concept of quality in education:a review of the "international" literature on the concept of quality in education. England: EdQual, 2006.

BARTON, Keith C. ; LEVSTIK, Linda S. Teaching history for the common good. New York: Routledge, 2004.

CAMARGO, Siddharta. La evaluación del espacio histórico en la prueba ENLACE 2010. Perfiles Educativos. México, v. 34, n. 137, p. 10-27, 2012.

CARRETERO, Maria; POZO, Juan Ignacio; ASENSIO, M Mikel. (Comps.). La enseñanza de las ciencias sociales. Madrid: Visor, 1989.

CARRETERO, Mario Comprensión y aprendizaje de la historia. In: Enseñanza y aprendizaje de la historia en Educación Básica, México: SECRETARÍA DE EDUCACIÓN PÚBLICA, 2011, p. 73-108.

COMISIÓN ECONÓMICA PARA AMÉRICA LATINA Y EL CARIBE y ORGANIZACIÓN DE NACIONES UNIDAS PARA LA EDUCACIÓN, LA CIENCIA Y LA CULTURA. Educación y conocimiento: eje de la transformación productiva con equidad. Santiago de Chile: COMISIÓN ECONÓMICA PARA AMÉRICA LATINA Y EL CARIBE Y ORGANIZACIÓN DE NACIONES UNIDAS PARA LA EDUCACIÓN, 1992.

LAMONEDA, Mireya. ¿Cómo enseñamos historia en el nivel medio superior?. Revista Mexicana de Investigación Educativa, México, v.3, n. 5, p. 101-112, 1998.

LAMONEDA, Mireya. Una alternativa en el enseñanza de la historia a nivel primaria. México: CIESAS: Cuadernos de las Casa Chata, 1990.

LEE, Peter; ASHBY, Rosalyn. Progression in Historical Understanding amon Students Ages 7-14. In: STEARNS, Peter; SEIXAS, Peter; WINEBURG, Samuel. Knowing, teaching \& leraning history: national and international perspectives. New York: New York University Press, 2000.

LERNER, Victoria. Libros de historia para niños:parámetros y dificultades para elaborarlos. Perfiles Educativos, México, n. 62, p. 49-55, 1993. 
LERNER, Victoria. Propuestas para el desarrollo de la didáctica de la Historia de México. Perfiles Educativos. México, n. 67, p. 18-26, 1995

LIMA. Laura; BONILLA, Felipe; ARISTA, Verónica. La enseñanza de la historia en la escuela mexicana. Proyecto Clío 36: Barcelona, 2010, Disponible en <http://clio.rediris.es>, Consultado: 18/02/2013

LIMA, Laura. La enseñanza de la historia, a debate. Educar. Revista de Educación: Guadalajara, México, 2005p. 87-90.

MÉXICO.Compromiso Social por la calidad de la educación, 2002. Disponible en <http://transparencia.yucatan.gob.mx/datos/2008/sep/marco_Juridico/Compr_Soc_Cal_E duc.pdf >, Consultada el 05/02/2014

MÉXICO. Plan Nacional de Educación 2001-2006:por una educación de buena calidad para todos. Un enfoque educativo para el siglo XXI, 2001, Disponible en <http://planipolis.iiep.unesco.org/upload/Mexico/Mexico\%20Programa\%2onacional\%20de\% 20educacion\%202001-2006.pdf>. Consultado el 9/12/2013

MÉXICO. Programa Sectorial de Educación 2007-2012, 2007. Disponible en: <http://basica.sep.gob.mx/dgme/pdf/cominterna/ProgramaSectorlal20072012.pdf >.Consultado el 9/12/2013

O'GORMAN, Edmundo. Crisis y porvenir de la ciencia histórica. México: Prensa Universitaria, 1947.

PAGÉS, Joan. Aproximaciones a un currículum sobre el tiempo histórico. IN: RODRíGUEZ FRUTOS, et al. Enseñar historia: nuevas propuestas. México: Fontamara, 2002, p. 109-140

PLÁ, Sebastián. ¿Sabemos historia en educación básica? una mirada a los resultados de ENLACE 2010. Perfiles EducativosMéxico,n. 134, pp. 138-154, 2011.

PLÁ, Sebastián.Desplazamientos en la didáctica de la historia o la transposición psicológica de una asignatura escolar. In: Reinalda Soriano Peña y Ma. Dolores Ávalos Lozano (coord.) Análisis político de discurso:dispositivos intelectualesen la investigación social. México: Juan Pablos, 2009, p. 313-327.

PLÁ, Sebastián. La enseñanza de la historia en México: o la fabricación del 'último mexicano' (1993-2011). Historiografías: Revista de historia y teoría, España, n. 4, p. 47-61, 2012.

PLÁ, Sebastián. Metamorfosis del discurso histórico escolar. Revista Teoría y Didáctica de las Ciencias Sociales, Caracas: Universidad de los Andes, n. 13, p.171-194, 2008. 
POY, Laura. Enlace sólo se usó para premiar o castigar a docentes: especialistas. la jornada, México, 2 de febrero de 2014.

SECRETARÍA DE EDUCACIÓN PÚBLICA, Acuerdo 592 por el que se establece la articulación a la Educación Básica. México: SECRETARÍA DE EDUCACIÓN PÚBLICA 2011.

SECRETARÍA DE EDUCACIÓN PÚBLICA.Decreto por el que se crea el Instituto Nacional para la Evaluación de la Educación. 2002. Disponible en

<http://www.inee.edu.mx/index.php/comite-de-validez/cat_view/17-documentos-delinee/18-normateca/1-normas-internas-del-inee/4-normas-sustantivas-del-inee/5presidencia-del-inee>; Consultada el 05/02/2014

SECRETARÍA DE EDUCACIÓN PÚBLICA. Programas de Estudio 2011:guía para el maestro. Educación Básica. Secundaria. México: SECRETARÍA DE EDUCACIÓN PÚBLICA, 2011a.

SECRETARÍA DE EDUCACIÓN PÚBLICA. Educación secundaria: Historia, programas de estudio. .México: SECRETARÍA DE EDUCACIÓN PÚBLICA, 2006.

SECRETARÍA DE EDUCACIÓN PÚBLICA. Reforma Integral de la educaicón básica: planes y programas de estudio de 1993 y 2009 (puntos de continuidad y/o cambio). México: SECRETARÍA DE EDUCACIÓN PÚBLICA, 2009.

SEIXAS, Peter; PECK, Carla. Benchmarcks of historical thinking. First stops. Canadian Journal of Education: Canada,v. 31, n 4, p. 1015-1038, 2008.

SHULMAN, Lee. Conocimiento y enseñanza: fundamentos de la nueva reforma. Profesorado. Revista de currículum y formación del profesorado, v. 9, n. 2, p. 1-30, 2005.

SOUSA SANTOS, Boaventura. Refundación del Estado en América Latina. Perspectivas desde una epistemología del sur. Lima: Instituto Internacional de Derecho y SociedadPrograma Democracia y Transformación Social, 2010.

ORGANIZACIÓN DE NACIONES UNIDAS PARA LA EDUCACIÓN, LA CIENCIA Y LA CULTURA. Declaración Mundial sobre educación para todos y marco de acción para satisfacer las necesidades básicas de aprendizaje. Jomtien, Tailandia: UNESCO, 1990.

WINEBURG, Samuel. Historical thinking and other unnatural acts:charting the future of teaching the past. Estados Unidos:Temple University Press, 2001.

WINEBURG, Sam. The psychology of Learning and teaching history. In: BERLINER, D. ; CALFEE, R. (Eds.). Handbook of educational psychology. New York: Simon \& Schuster Macmillan, 1996, p. 423-437. 
Recebido em: 10/01/2014 Aprovado em: 10/03/2014

Universidade do Estado de Santa Catarina - UDESC Programa de Pós-Graduação em História - PPGH

Revista Tempo e Argumento Volume 06 - Número 11 - Ano 2014 tempoeargumento@gmail.com 